\title{
Dramatic Repair of Joint Damage in Psoriatic Arthritis
}

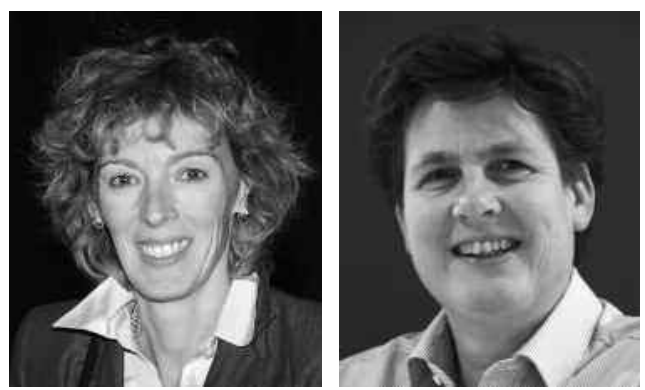

Repair on radiographs of hands and feet is not a new phenomenon. Carefully comparing consecutive sets of radiographs of patients with rheumatoid arthritis (RA) in clinical remission occasionally revealed disappearing erosions over time in the prebiological era ${ }^{1}$, but repair, or "healing" as it was called in those days, was not systematically evaluated until recently. Nevertheless, there were true believers of healing that rightfully claimed, as do Eder and colleagues in this issue of The Journal in patients with psoriatic arthritis $(\mathrm{PsA})^{2}$ : "We believe what we see."

The methodological interest in repair was fuelled by the advent of phase 3 trials with tumor necrosis factor (TNF) inhibitors. These trials showed for the first time in history not only a near-complete arrest in radiographic progression, but also negative progression scores in a significant proportion of the patients treated with TNF inhibitors. In order to understand these negative scores, it is important to briefly outline how progression scores are generated in clinical trials.

Usually, 2 independent readers are presented with consecutive sets of radiographs of hands and feet in order to comparatively score these radiographs on a per-joint basis for erosions and joint space narrowing. Importantly, these readers are not aware of the true time order of the radiographs, so that they cannot interpret the changes they may see. All joint change scores are then summed up, and the total sum score of a patient is reported as the change score. Such a change score can be negative, and it is tempting to consider a negative score as a proof of repair in that patient.

But there is more to take into consideration. Scoring radiographs is a human and "manual" task, often performed on all but optimal radiographs that are also often not exactly similar, which makes this methodology prone to measurement error. Theoretically, measurement error is symmetrical in the positive and negative directions as long as blinding of true time order is strictly preserved, and we do see this symmetry in probability plots of clinical trials with TNF blockers. The signal of true progression or true repair, how- ever, is mixed up with the signal of measurement error, and can impossibly be disentangled and quantified. To further complicate the matter, it is known that on a per-joint basis negative change scores and positive change scores may occur in one and the same patient, and obviously, the direction of the final sum score is determined by the balance of negative and positive scores. The same is true for the entire trial population that may consist of patients with a positive and those with a negative score. So, altogether it seems impossible to scientifically prove that repair is present in a patient, a clinical trial, or a population of patients with a condition like RA or PsA.

Fortunately, there is one exception, and we have indeed seen that exception in the recent past. If a population of patients in a trial shows a negative mean progression score, that negative mean progression score is statistically different from zero (no change), and the scores are obtained in a truly unbiased manner (blind time order), one may claim that repair in that group overrules progression, even if the latter co-exists.

This is exactly what was found in a few trials with TNF blockers in RA: the TEMPO trial ${ }^{3}$ and the RAPID 2 trial $^{4}$ - a statistically significant negative progression score in the group of patients treated with a TNF inhibitor. After further exploration on a per-joint basis, we have learned that the negative joint scores were preferably seen in joints that were not active (any more), and in patients that used etanercept in combination with methotrexate ${ }^{5}$. Meanwhile, an OMERACT (Outcome Measures in Rheumatology Clinical Trials) initiative on repair in RA concluded - somewhat against the expectations of the "believers" - that repair could not be distinguished from progression by using specific repair features, but repair was more or less the reverse of progression ${ }^{6}$. This implies, for example, that erosions may "fill in" over time and form new cortex.

So, how should the finding by Eder, et al be interpreted? Do they truly demonstrate repair in their patient with PsA? A few remarks should be made. First, in light of experience

See Repair of radiographic joint damage after etanercept in PsA, page 1066

Personal non-commercial use only. The Journal of Rheumatology Copyright (c) 2011. All rights reserved. 
with TNF inhibitors in RA and similarities in pathophysiology in RA and PsA with regard to bone formation and degradation, there is a rather high prior likelihood that repair can occasionally be found in patients with PsA treated with TNF-blocking drugs. Second, Eder, et al show a remarkable example of a patient in which the total Sharp-van der Heijde score decreases from 59 in 2005 to 26 in 2009, which we have probably never seen in RA. Undoubtedly, part of this remarkable effect is due to measurement error and expectation bias, but looking at the successive radiographs, no one can deny there is dramatic improvement over time. However, such patients must be very rare. Third, radiographic features of PsA and RA differ with respect to distal interphalangeal joint involvement, a tendency to bony ankylosis and bony proliferation. Interestingly, Eder, et al quantified proliferation in their patient according to the Ratingen score, and found almost no effect on proliferation, while the erosion score was dramatically reduced over time, an observation that is in line with trial observations in PsA. Another disease in which bone proliferation is a characteristic feature is ankylosing spondylitis (AS). Also in AS, the formation and growth of syndesmophytes, due to bony proliferation, are insensitive to the effects of TNF blockade ${ }^{7}$, which may point to shared pathophysiological mechanisms in bone metabolism in both diseases. It can also be hypothesized that the massive reduction in erosions seen in this patient with PsA is related to bony proliferation, which makes it "easier" in PsA for erosions to fill in, compared to RA, where such a bony proliferation does not exist.

In summary, Eder, et al have presented an interesting report of a patient with PsA and a dramatic improvement in erosion scores under the influence of etanercept. In light of findings in RA, repair in PsA is highly likely, but will rarely, if ever, be found at such a level as presented here. The scientific quantification of repair at the trial level requires a far subtler approach in which nonspecific effects often overrule specific repair.
DÉSIRÉE van der HEIJDE, $\mathrm{MD}, \mathrm{PhD}$,

Department of Rheumatology,

Leiden University Medical Center,

PO Box 9600, 2300 RC Leiden,

The Netherlands;

ROBERT B.M. LANDEWÉ, $M D, P h D$,

Academic Medical Center/University of Amsterdam, and Atrium Medical Center Heerlen,

Heerlen, The Netherlands

Address correspondence to Dr. van der Heijde;

E-mail:d.vanderheijde@kpnplanet.nl

\section{REFERENCES}

1. Rau R, Herborn G. Healing phenomena of erosive changes in rheumatoid arthritis patients undergoing disease-modifying antirheumatic drug therapy. Arthritis Rheum 1996;39:162-8.

2. Eder L, Chandran V, Gladman DD. Repair of radiographic joint damage following treatment with etanercept in psoriatic arthritis is demonstrable by 3 radiographic methods. J Rheumatol 2011;38:1066-70

3. Klareskog L, van der Heijde D, de Jager JP, Gough A, Kalden J, Malaise M, et al. Therapeutic effect of the combination of etanercept and methotrexate compared with each treatment alone in patients with rheumatoid arthritis: double-blind randomised controlled trial. Lancet 2004;363:675-81.

4. Smolen J, Landewe RB, Mease P, Brzezicki J, Mason D, Luijtens $\mathrm{K}$, et al. Efficacy and safety of certolizumab pegol plus methotrexate in active rheumatoid arthritis: the RAPID 2 study. A randomised controlled trial. Ann Rheum Dis 2009;68:797-804.

5. Lukas C, van der Heijde D, Fatenajad S, Landewe R. Repair of erosions occurs almost exclusively in damaged joints without swelling. Ann Rheum Dis 2010;69:851-5.

6. van der Heijde D, Landewé R, Sharp JT. Repair in rheumatoid arthritis, current status. Report of a workshop at OMERACT 8 J Rheumatol 2007;34:884-8.

7. van der Heijde D, Landewé R, Einstein S, Ory P, Vosse D, Ni L, et al. Radiographic progression of ankylosing spondylitis after up to two years of treatment with etanercept. Arthritis Rheum 2008;58:1324-31.

J Rheumatol 2011;38:969-70; doi:10.3899/jrheum.110196 